\title{
POORER Q GIVES BETTER MASS SENSITIVITY: RECONSIDERING DYNAMIC RANGE IN OPTOMECHANICALY TRANSDUCED NEMS DEVICES
}

W.K. Hiebert ${ }^{1,2^{*}}$, S.K. Roy ${ }^{1,2}$, and V.T.K. Sauer ${ }^{1,2}$

${ }^{1}$ National Institute for Nanotechnology, NRC Canada, Edmonton, Alberta, Canada

${ }^{2}$ University of Alberta, Edmonton, Alberta, Canada

\begin{abstract}
We demonstrate that reduced mechanical quality factor can lead to improved frequency stability and mass sensitivity in a nano-optomechanical device. We measure the dynamic range and Allan deviation of frequency fluctuations in a phase-locked NEMS device at different pressures and quality factors, including at atmospheric pressure. As quality factor decreases, dynamic range increases and Allan deviation improves. Key to this improvement is that the dynamic range in vacuum and in 6 Torr pressure is limited only by the mechanical Duffing nonlinearity and the thermomechanical noise floor. This allows a fractional frequency stability of part per billion and a mass sensitivity in the zeptogram range for operation in 6 Torr pressure. These results open the door toward use of ultrasensitive NEMS devices in gas, liquid, and other low-Q environments.
\end{abstract}

\section{INTRODUCTION}

NEMS are well-known for achieving incredible results in terms of mass sensitivity with single molecule [1], even single Da sensitivity levels [2,3]. Transducing these tiny devices at all, let alone taking advantage of the full dynamic range (DR), is an extraordinary challenge [4]. Optomechanical transduction [5] has recently been shown to be very promising for conserving DR while size-down-scaling [6]. Particularly, it offers exquisite displacement sensitivity to nanomechanical motions, reaching into the attogram per root Hertz range [7]. This sensitivity level allows easily achieving thermal noise limited operation in a wide variety of conditions (high Q or low Q) and device size regimes. Further, the design freedom to tune optomechanical coupling strength allows full control over the linear operation range of the transduction. Together, these attributes make optomechanical readout perfectly suited for accessing the full intrinsic DR of NEMS devices.

This full intrinsic DR has seldom been accessed in state-ofthe-art sized NEMS devices of sub-micron cross-sectional dimensions and few micron lengths. In such devices, while the upper end of the DR is often accessible (represented by the Duffing nonlinearity) [8], normally, the noise floor is set by instrumentation noise levels [4]. These levels remain unchanged during device size down-scaling or changes in Q-factor and pose a serious problem for transducing small, low-Q NEMS mechanical resonances. As mentioned above, this problem is obviated when transducing with optomechanics.

Using the platform of state-of-the-art optomechanical NEMS devices, we look at the dependence of DR and mass sensitivity on mechanical quality factor (Q) and measurement bandwidth. We find the surprising result that poorer mechanical quality factor leads to better mass sensitivity when the intrinsic DR is maximized. We confirm this experimental finding in deriving the $\mathrm{DR}$ dependence on $\mathrm{Q}$. This result opens the door to ultrasensitive sensing in gas, liquid, or other low-Q environments.

\section{BACKGROUND AND THEORY}

The conventional picture of improving sensitivity with resonant micromechanical devices and the importance of DR was discussed in [4] (see Figure 1). In an ideal case, the resonator is driven close to a nonlinearity limit, such a Duffing nonlinearity

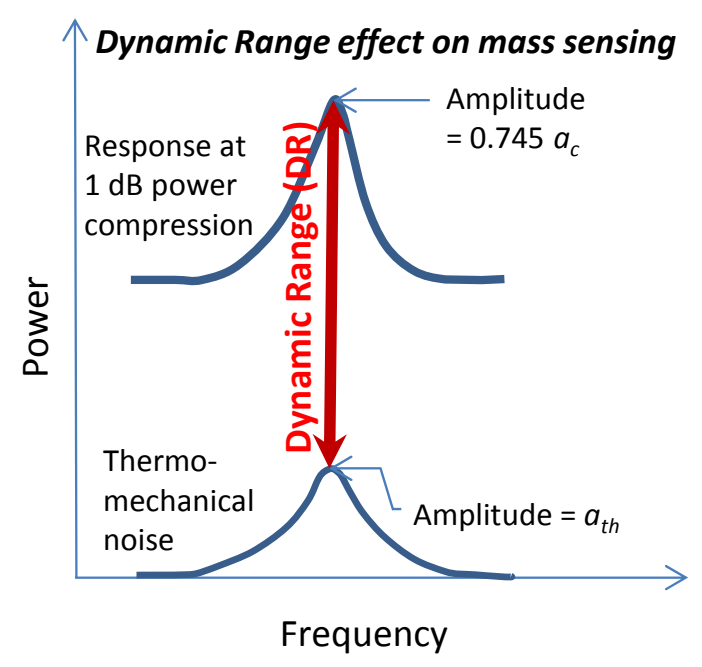

Figure 1. The intrinsic dynamic range of a resonant nanomechanical system. Upper limit is set by the onset of nonlinearity. Lower limit is set by the thermomechanical noise.

(specifically, to an amplitude where $1 \mathrm{~dB}$ power compression is observed) and the thermomechanical (TM) noise is resolvable. The DR represents the $\mathrm{dB}$ difference in power levels of these two extremes. The equation for the rms critical amplitude $a_{c}$ at the top end of the range in doubly clamped beams is [9]:

$$
a_{c}=\frac{\sqrt{2} d}{\sqrt{3 Q \sqrt{3}}}
$$

where $d$ is the thickness of the NEMS device in direction of motion and $\mathrm{Q}$ is the mechanical quality factor. The equation for the amplitude at the bottom end of the range $a_{t h}$ is the thermal noise:

$$
a_{t h}=S_{X}{ }^{1 / 2} \Delta f^{1 / 2}=\left(\frac{4 k_{B} T Q \Delta f}{M_{e f f} \omega_{0}^{3}}\right)^{1 / 2}
$$

where $k_{b}$ is Boltzmann's constant, $T$ is the Temperature, $\Delta f$ is measurement bandwidth, $M_{\text {eff }}$ is the effective mass of the resonator, and $\omega_{0}$ is $2 \pi$ times the resonance frequency $f$. The DR in $\mathrm{dB}$ is defined explicitly as:

$$
D R(d B)=20 \log \left(\frac{0.745 a_{c}}{a_{t h}}\right)
$$

The fractional frequency stability $\delta / f f$ (and mass sensitivity) improves when increasing DR according to the following relationship [4]:

$$
\frac{1}{2} \frac{\delta m}{M_{e f f}}=\frac{\delta f}{f} \approx\left(\frac{\Delta f}{Q \omega_{0}}\right)^{1 / 2} 10^{(-D R / 20)}
$$

A closer examination of equations (1) to (4) reveals a very intriguing relationship. For the upper end of the DR the Duffing nonlinearity leads to a $Q^{-1 / 2}$ dependence of the critical amplitude (from (1)). On the bottom end of the DR, the displacement noise leads to a $Q^{+1 / 2}$ dependence (from (2)). Together, these relationships imply that the $D R$ can be increased at both ends by reducing the quality factor of the resonator. Explicitly, if mass, 
temperature, and resonance frequency remain the same, substituting (1) and (2) into (3) implies:

$$
D R(d B)=20 \log \left(\frac{\xi}{Q \Delta f^{1 / 2}}\right)
$$

where $\xi$ is a constant. In essence, as long as more drive power is available, a poorer $\mathrm{Q}$ device can be driven to larger amplitude than a better $\mathrm{Q}$ one can. Similarly, the TM noise on resonance is suppressed in the poorer Q device compared with the better Q one, and this can be taken advantage of as long as enough transduction sensitivity is available to resolve the lower noise floor. When applying these findings to the $\delta f / f$ (or mass sensitivity) relationship (equation (4)), one finds that:

$$
\delta m \propto Q^{1 / 2} \Delta f
$$

Amazingly, the mass sensitivity level becomes smaller (better) for poorer $Q$.

\section{DEVICE FABRICATION AND EXPERIMENT}

A racetrack resonator optical cavity structure is used to detect the nanomechanical motion of the doubly-clamped beam [10]. The $220 \mathrm{~nm}$ Silicon on Insulator devices are fabricated by a photonic foundry (IMEC) and NEMS devices are released by postprocessing at the University of Alberta Nanofab. The NEMS are designed to be narrower than the photonic lines, facilitating a maskless release step wherein the NEMS are released without releasing the photonic elements. The NEMS are coupled laterally to the photonic elements and are contained in the same device layer. Further description of the fabrication can be found in [10]. A SEM picture of the device studied in this paper is shown in Fig. 2(a).

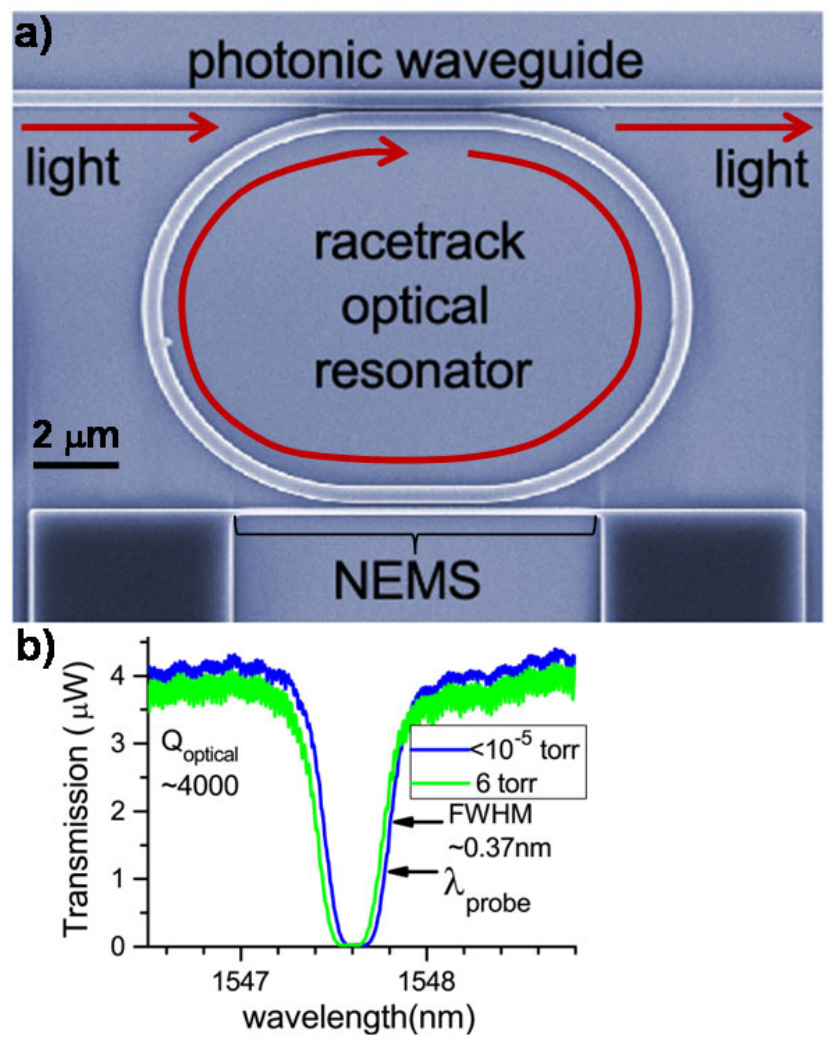

Figure 2. (a) Annotated scanning electron micrograph of the nanooptomechanical device of dimensions $8.75 \mu \mathrm{m} \times 160 \mathrm{~nm} \times 220 \mathrm{~nm}$. The gap between racetrack and NEMS is $130 \mathrm{~nm}$. (b) Optical resonance at high vacuum and 6 Torr. The probe wavelength sits at a large slope in the transmission vs wavelength in order to transduce NEMS vibrations into optical power modulations.
The devices are driven by a sheer piezo actuating the chip and interrogated using a free-space confocal lens system to couple light to on-chip grating couplers [11]. NEMS motions are transduced by their interaction with the evanescent field of the light in the optical racetrack. Essentially, NEMS motion modulates the wavelength of the optical cavity resonance. Setting a laser probe wavelength on the steep side slope of an optical resonance translates that modulation into optical transmission power modulation (see Fig. 2(b)). This optical power modulation is read out by photodiode to ultimately give a voltage modulation that is directly proportional to the NEMS displacement. Further details of the experiment can be found in [6], [10], and [11].

\section{RESULTS}

In order to demonstrate the dependence of the dynamic range on Q (equation (5)), we measure the properties of the same device at different pressures. In particular, as we change the pressure from high vacuum $\left(\sim 10^{-5}\right.$ Torr), to 6 Torr, to 760 Torr, the quality factor drops by two orders of magnitude. This method has the benefit of keeping all other parameters that could affect the result precisely identical. The primary results are displayed in Fig. 3.

At each pressure, the thermomechanical noise is measured and plotted on the same voltage axis (for a $1 \mathrm{~Hz}$ bandwidth). Of particular note is how the noise floor peak diminishes as the pressure increases (and the Q decreases). This is consistent with equation (2) and can be conceptually understood in the following way. The area under the TM resonance curve is conserved for a given Temperature. As the width of the resonance curve increases (i.e. $\mathrm{Q}$ decreases), the peak of the TM curve must drop in order to compensate. The TM curve is so wide by 760 Torr, it looks flat on the right most plot (the inset shows a wider field of view of the atmospheric TM noise). The reduction in $\mathrm{Q}$ from high vacuum to 6 Torr is about a factor of 3. This should imply a reduction of $\sqrt{3}$ in peak height. The actual peak height is reduced by a full factor of 3. Slight differences in displacement to voltage responsivity could explain part of this discrepancy. From 6 Torr to 760 Torr, $\mathrm{Q}_{\mathrm{TM}}$ drops by 40x. We thus expect a drop in peak height by about $6 \mathrm{x}$ and this is what is observed.

The upper portion of Fig. 3 shows frequency sweeps of the resonance with increasing powers in order to find the limit of nonlinearity. For vacuum and 6 Torr, the nonlinearity is consistent with a Duffing nonlinearity. The $1 \mathrm{~dB}$ compression curve is noted in bold (red) in each case and $a_{c r i t}=0.745 a_{c}$ is marked. Again, of note here is how the value for $a_{\text {crit }}$ increases moving from lower pressure to 6 Torr. A fit of Lorentzians to the critical sweep gives Q-factors for the two strongly driven cases. There are slight changes to the Q-factor in comparison to the TM noise derived Qs and the ratio is now around $2.4 \mathrm{x}$. We thus expect an increase in $a_{\text {crit }}$ of $\sqrt{2.4}$ or about $1.5 \mathrm{x}$, and this is roughly what is observed.

Overall, the Q effects on the ends of the linear range lead to a $4 \mathrm{x}$ increase in the linear range or a $12 \mathrm{~dB}$ increase in the DR from $54 \mathrm{~dB}$ to $66 \mathrm{~dB}$, when moving from high vacuum to 6 Torr.

The atmospheric pressure curve (right-hand side) of Fig. 3 has additional information to add to the story. The TM noise changes as expected compared to 6 Torr, however, the nonlinearity encountered is no longer a Duffing nonlinearity. Instead, a rather complex nonlinear response is evident at these very high drive powers in atmospheric pressure. More will be discussed about this in the context of Fig. 5. In any case, it is understandable that the trend of increasing $a_{\text {crit }}$ no longer continues since other nonlinearities come into play before Duffing is encountered. It is important to note that the DR at atmospheric pressure is still better than that at high vacuum. This bodes very well for devices operating as gas sensors at atmospheric pressure. 


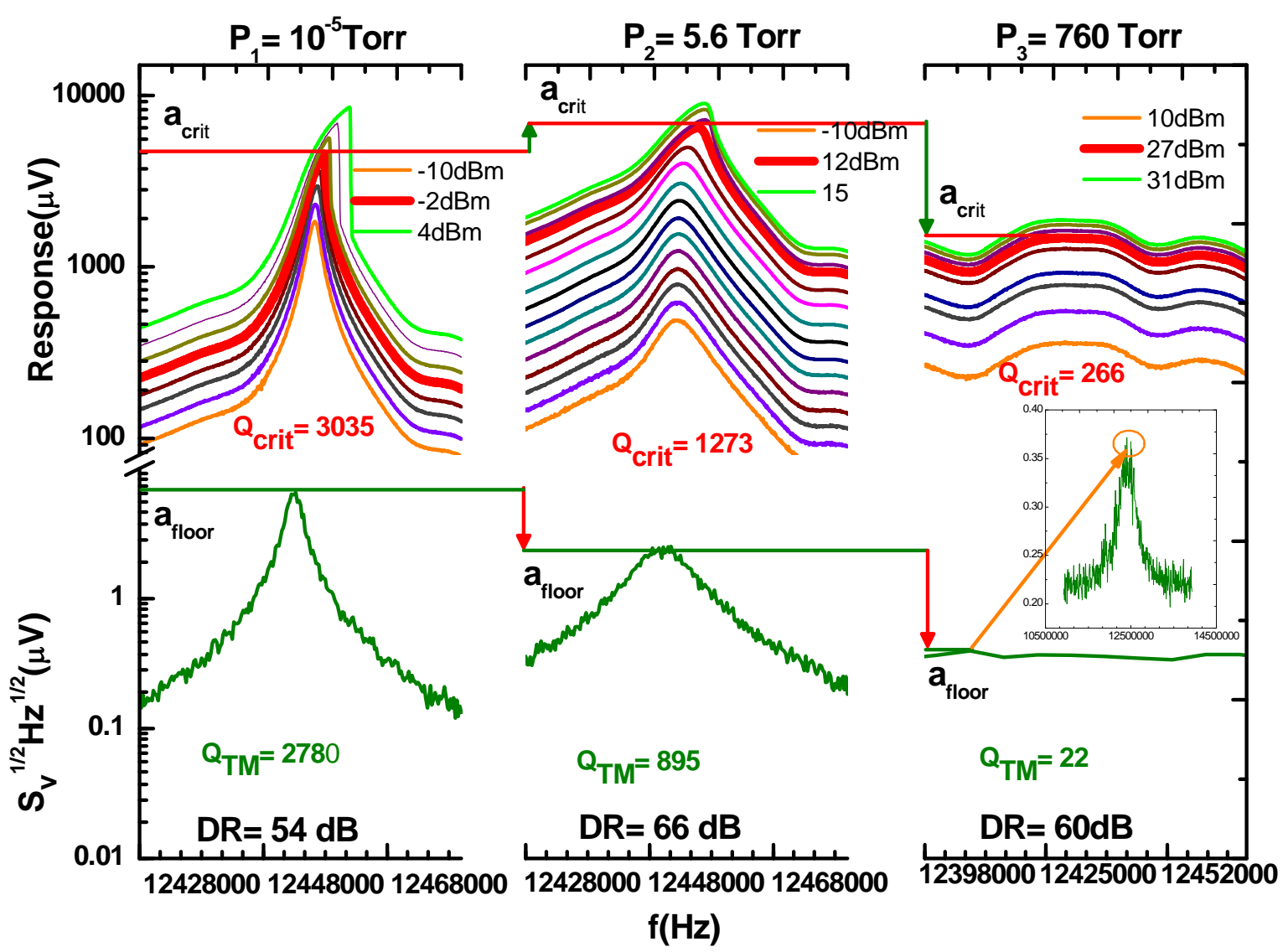

Figure 3. Summary of results on a single NEMS device operated at three different pressures (and different quality factors). Thermomechanical noise is resolved at small signal voltages in all three cases. The peak of the TM noise goes down with increasing pressure (decreasing Q). Responses to increasing drive powers are plotted at higher voltages. The thicker red plot highlights the responses for $1 \mathrm{~dB}$ signal compression and allows us to define $a_{\text {crit. }}$ Duffing nonlinearities are reached for high vacuum and 6 Torr; a pressure induced nonlinearity is reached at atmospheric pressure.

Figure 4 shows how the increase in DR translates into improvement in fractional frequency stability and mass sensitivity. Allan deviation as a function of sampling time $\tau$ is plotted for two pressures (two values of $\mathrm{Q}$ ) and two bandwidths. According to equation 6, we expect fluctuations to be proportional to both $\sqrt{Q}$ and bandwidth $\Delta f$. In both pressures the reduction of the bandwidth from $10 \mathrm{~Hz}$ to $2 \mathrm{~Hz}$ does indeed lead to a drop in the Allan deviation of approximately a factor of 5 as expected. The drop in Allan deviation of the right graph with respect to the left is roughly a factor of $2.5 \mathrm{x}$. This is slightly more than the factor of $\sqrt{3}$ as expected from equation 6 , but it is in the right expected direction and rough magnitude, and clearly demonstrates better performance in the lower Q case. This is an exciting development that could lead to better performance of NEMS mass sensors in gaseous environments. An estimation of the Allan deviation can also be made from equation (4). For $2 \mathrm{~Hz}, \mathrm{DR} \sim 54 \mathrm{~dB}$, and $\mathrm{Q} \sim 3000$, a value of $6 \times 10^{-9}$ is estimated. This is within a factor of 2 of the measured deviation at $\tau=0.5 \mathrm{~s}$.

Furthermore, the mass sensitivity can be estimated from equation (4) with knowledge of the effective mass of the NEMS device. A mass axis has been added to the right side of the figure accordingly. The roughly part per billion value of the frequency stability at 5.6 Torr and $2 \mathrm{~Hz}$ bandwidth translates into a $1 \mathrm{zg}$ noise level for the mass sensitivity of this particular device. This is state-of-the-art sensitivity for a top-down fabricated device, and is

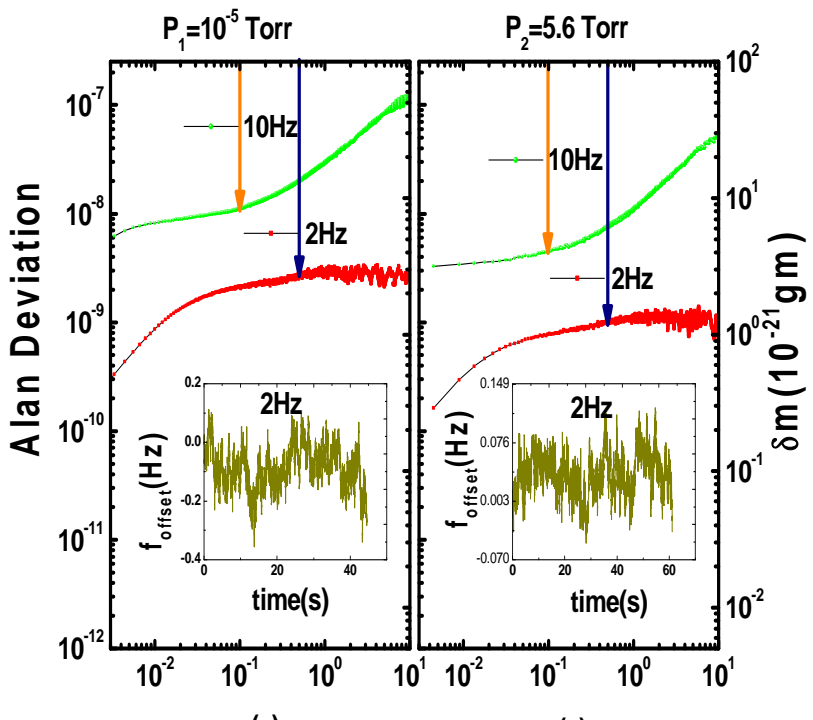

$\tau(\mathbf{s})$

$\tau(\mathbf{s})$

Figure 4. Allan deviation of NEMS frequency fluctuations at two different pressures and two different loop bandwidths. Insets: frequency fluctuations vs time for the $2 \mathrm{~Hz}$ cases. The low $\mathrm{Q}$ case (higher pressures) has better Allan deviation and mass sensitivity. 
a state-of-the-art demonstration for any device subjected to gas damping.

Finally, we return to the prospect of atmospheric pressure sensing of mass by further investigating the atmospheric response in Fig. 5. From Fig. 3, $\mathrm{Q}_{\text {crit }}$ at 760 torr is 16 times higher than $\mathrm{Q}_{\mathrm{TM}}$ at the same pressure. We elucidate this discrepancy by showing a $1 \mathrm{MHz}$ frequency span of the driven resonance mode in the right hand side of Fig. 5 for $2 \mathrm{~V}$ driving power. Several peaks are evident around a highest central peak, each with its own phase change, the whole roughly taking on an envelope similar to the TM noise curve shape; it is as if the wider resonance peak has split into several, narrower, independent peaks. It was found that the position and shape of these peaks with their associated phases remains the same throughout all tested driving voltages, including up to $7 \mathrm{~V}$. Furthermore each of the narrower peaks can be easily phase locked. From the phase data, we can estimate $\mathrm{Q}$ values by using the equation $\frac{d \emptyset}{d f}=\frac{2 Q}{f_{\text {resonance }}}$. The left part of Fig. 5 has $\mathrm{Q}$ calculated for the critical driving voltage in this way, as well as by Lorentz fitting the tallest peak. Both Q values (277, and 369) are similar and give us confidence in our assigning of an effective Q value of $\mathrm{Q}_{\text {crit }}=366$ in Fig. 3.

We do not yet fully understand this partitioning of the atmospheric pressure peak into narrower pieces, but we do note that it could be used to advantage by allowing phase locking to a resonance that is essentially an order of magnitude sharper than would otherwise be the case.

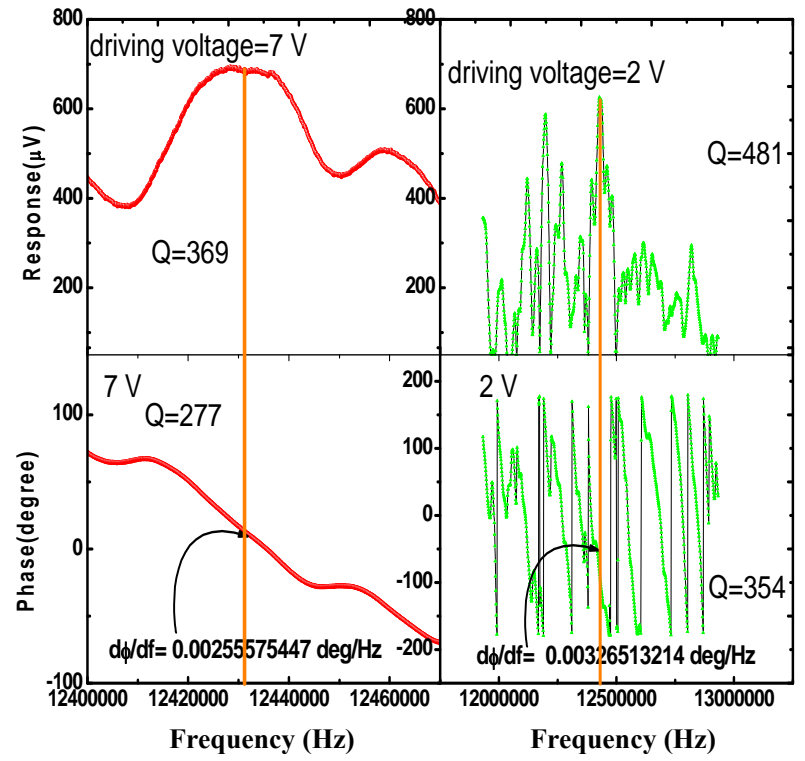

Figure 5: Magnitude and phase response of the device at 760 Torr for $7 \mathrm{~V}$ (critical driving voltage, $+27 \mathrm{dBm}$ ) (left side) and for $2 \mathrm{~V}$ (right side). The right side shows several peaks within a larger envelope over the $1 \mathrm{MHz}$ span, each with a distinctive resonance phase change. The left side shows the consistency of assigning a high effective quality factor to a narrower resonance. Orange line is a guide for eye to show the quality factor calculated at center frequency.

\section{CONCLUSIONS}

We have used optomechanical transduction to investigate the full intrinsic dynamic range of a nano-optomechanical device at vacuum, 6 Torr, and atmospheric pressures. We looked at the dependence of dynamic range on quality factor and both measured and derived a relationship that sees the intrinsic DR increase as $\mathrm{Q}$ decreases, demonstrating DR of $54 \mathrm{~dB}, 66 \mathrm{~dB}$ and $60 \mathrm{~dB}$ at vacuum 6 , Torr, and atmospheric pressure, respectively. Further, we have shown that reduction of $\mathrm{Q}$ leads to improved frequency stability and mass sensitivity and demonstrated a record zeptogram sensitivity level at 6 Torr pressure. Finally, we have shown an order of magnitude increase in effective quality factor at atmospheric pressure as well as an improvement in dynamic range compared to vacuum. These findings hold promise for use of resonant mechanical sensors in gas and liquid environments and also open the door to increased usage of any types of resonant mechanical systems in more diverse surroundings.

\section{ACKNOWLEDGEMENTS}

This work was supported by the National Institute for Nanotechnology (NINT), Alberta Innovates Technology Futures, the Natural Sciences and Engineering Research Council, Canada, the Canadian Institute for Advanced Research, and CMC Microsystems. The fabrication of the devices was facilitated through CMC Microsystems, and post processing was done at the University of Alberta NanoFab. Simulation software was provided by the Integrated Nanosystem Research Facility at the University of Alberta. SEMs were taken at the Electron Microscopy Facility at NINT.

\section{REFERENCES}

[1] A. K. Naik, M. S. Hanay, W. K. Hiebert, X. L. Feng, and M. L. Roukes, "Towards single-molecule nanomechanical mass spectrometry," Nature Nanotechnology 4, 445 (2009).

[2] J. Chaste, A. Eicher, J. Moser, G. Ceballos, R. Rurali, and A. Bachtold, "A nanomechanical mass sensor with yoctogram resolution," Nature Nanotechnology 7, 301 (2012).

[3] W. K. Hiebert, "Mass sensing Devices reach single proton limit," Nature Nanotechnology, 7, 278 (2012).

[4] K. L. Ekinci, Y. T. Yang, and M. L. Roukes, "Ultimate limits to inertial mass sensing based upon nanoelectromechanical systems," J. App. Phys. 95, 2682 (2004).

[5] M. Li, W. Pernice, C. Xiong, T. Baehr-Jones, M. Hochberg, and H. Tang, "Harnessing optical forces in integrated photonic circuits," Nature 456, 480 (2009).

[6] V. T. K. Sauer, Z. Diao, M. R. Freeman, and W. K. Hiebert, "Optical racetrack resonator transduction of nanomechanical cantilevers," Nanotechnology 25, 055202 (2014).

[7] M. Eichenfield, R. Camacho, J. Chan, K, J. Vahala, and O. Painter, "A picogram- and nanometer-scale photonic-crystal optomechanical cavity," Nature 444, 67 (2009).

[8] X C. Zhang, E. B. Myers, J. E. Sader, and M. L. Roukes, "Nanomechanical torsional resonators for frequency-shit infrared thermal sensing," Nano Letters 13, 1528 (2013).

[9] H. W. Ch. Postma, I. Kozinsky, A. Husain, and M. L. Roukes, "Dynamic range of nanotube- and nanowire-based electromechanical systems," Appl. Phys. Lett. 86, 223105 (2005).

[10] V. T. K. Sauer, Z. Diao, M. R. Freeman, and W. K. Hiebert, "Nanophotonic detection of side-coupled nanomechanical cantilevers," Appl. Phys. Lett. 100, 261102 (2012).

[11] Z. Diao, J. E. Losby, V. T. K. Sauer, J. N. Westwood, M. R. Freeman, and W. K. Hiebert, "Confocal scanner for highly sensitive photonic transduction of nanomechanical resonators," Appl. Phys. Express 6, 065202 (2013).

\section{CONTACT}

*W.K. Hiebert, tel: +1-780-641-1708; Wayne.Hiebert@nrc.ca 